\title{
Les annuaires hydrologiques de la Société hydrotechnique de France : une source d'information patrimoniale pour la connaissance de l'hydrologie en France
}

\author{
Chloé LE GROS ${ }^{1}$, Eric SAUQUET ${ }^{1}$, Michel LANG ${ }^{1}$, Anne-Laure ACHARD², \\ Etienne LEBLOIS $^{1}$, Brigitte BITON ${ }^{3}$
}

\author{
Irstea, HHLY, 5 rue de la Doua CS70077, 69626 Villeurbanne, France - eric.sauquet@irstea.fr \\ 2. Irstea, IST, 5 rue de la Doua CS70077, 69626 Villeurbanne \\ SHF, 25 Rue des Favorites, 75015 Paris, France
}

\begin{abstract}
RÉSUMÉ. - La Société Hydrotechnique de France a publié entre 1939 et 1969 une série d’annuaires hydrologiques incluant, entre autres, des observations de débits sur un ensemble de stations hydrométriques françaises et des contributions techniques ouvertes sur des sujets relatifs à l'hydrologie. Longtemps disponible sur support papier uniquement, le contenu de ces annuaires a été numérisé afin d'être porté à la connaissance de la communauté hydrologique. Les objectifs de cet article sont de préciser le contenu de ces annuaires, de partager l'expérience d'extraction des séries numériques et d'identifier les données complémentaires qui pourraient venir enrichir les connaissances apportées par la banque HYDRO.
\end{abstract}

Mots-clés : numérisation d’archives, sauvetage de données, données anciennes, débit

\section{The hydrological yearbooks published by the Société Hydrotechnique de France: a valuable source of information on hydrology in France}

\begin{abstract}
The Société Hydrotechnique de France has published a series of hydrological yearbooks between 1939 and 1969 including daily flows observed at a subset of French gauging stations and technical contributions on several topics related to hydrology. The exact contents are not well-known and data available in the tables have not been digitized. The objectives of this article are to present the content of the hydrological yearbooks and the way time series were extracted and to identify additional historical data that may enrich the HYDRO database.
\end{abstract}

Key-words: digital processing, data rescue, historic data, river flow

\section{INTRODUCTION}

Aujourd’hui, la connaissance des débits en France s’appuie principalement sur le réseau hydrométrique qui alimente la banque de données HYDRO (http://hydro.eaufrance.fr). La majorité des séries hydrologiques bancarisées (près de 85 \%) débutent dans les années 1960 et un nombre réduit présente des enregistrements antérieurs à 1900. Or de longues séries sont requises notamment pour un calcul robuste des valeurs extrêmes (Lang et al., 2014), pour l'examen de la stationnarité des régimes hydrologiques (Giuntoli et al., 2013)...

Une manière de parfaire nos connaissances est de rechercher et d'exhumer des données anciennes renseignées dans des rapports techniques des services hydrométriques ou des retours d'expérience post-événements. Les Annuaires Hydrologiques de la Société Hydrotechnique de France font partie de ces sources archivées, peu accessibles par la communauté hydrologique. Edités de 1939 à 1969 par la Société Hydrotechnique de France (SHF), ils fournissent entre autres une synthèse de l'année écoulée au travers notamment de tableaux de débits journaliers et d'hydrographes annuels observés ou reconstitués sur un jeu de stations hydrométriques en fonctionnement. Ces annuaires n'étaient pas jusqu'à ce jour sur support informatique. Irstea (centre Lyon-Villeurbanne) et la SHF ont conjointement souhaité numériser leur contenu, pour porter ces documents à la connaissance de la communauté hydrologique, et, à terme, mettre à disposition les séries numériques qu'ils contiennent.

L'objectif de cet article est de décrire le contenu historique et patrimonial des annuaires, de partager notre expérience en matière d'extraction et de contrôle de données hydrologiques anciennes numérisées et de faire état des stations " inédites ", c’est-à-dire absentes à ce jour de la base de données HYDRO.

\section{PRESENTATION DES ANNUAIRES HYDROLOGIQUES DE LA FRANCE}

Si des premières mesures de cotes sur un nombre limité de cours d'eau existent de façon discontinue dans les années antérieures, c’est au cours de la seconde moitié du $\mathrm{XIX}^{\mathrm{e}}$ siècle que se met en place en France un réseau systématique d'observations hydrométriques avec la création des services d'annonce des crues, sous la direction des ingénieurs des Ponts et Chaussées. On commence à disposer sur un nombre significatif de cours d'eau d'informations 
sur la topographie des rivières (Nivellement Général de la France), sur les niveaux d'écoulement (relevés des services d'annonce des crues ; cotes de crues historiques), sur des mesures (premiers jaugeages) ou des estimations de débit (notes techniques). L'arrêté du ministre de l'Agriculture du 25 mars 1903 porte sur la création du Service des Grandes Forces Hydrauliques, pour l'évaluation du potentiel énergétique des cours d'eau montagnards français. La mission est officiellement organisée sur le secteur des Alpes, puis dix ans plus tard sur les Pyrénées et le Massif Central, sous la direction des ingénieurs du Génie Rural. Une série d'annuaires hydrologiques (Côte, 1908) va alors être publiée de 1911 à 1924 pour environ 200 stations de jaugeage. On trouve dans ces annuaires la carte de localisation des stations hydrométriques, le nivellement de cours d'eau sous forme de profils en long et le zéro altimétrique des échelles limnimétriques, le tableau des cotes et débits moyens journaliers, les jaugeages, le barème de tarage et la courbe de tarage, ainsi qu'un bilan des débits moyens mensuels et annuels.

La première guerre mondiale met un terme à la forte activité hydrométrique du début du XXe siècle. A l'issue de ce premier conflit, la France retrouve ses ressources minières du Nord et elle compte profiter de celles de l'Allemagne. Elle est trop profondément éprouvée pour maintenir des réseaux hydrométriques d'intérêt général. Ce n’est qu'en 1942 que l'Etat décide de reprendre la publication systématique des données hydrologiques, sous la forme d'annuaires.
Sous l'égide du Secrétariat d'Etat à la Production Industrielle (Direction de l'Electricité) et de la Société Hydrotechnique de France, ces annuaires seront édités pour les années 1939 à 1969, avec un décalage de 1 à 3 ans, le temps de collecter et critiquer les données.

Les annuaires vont comporter un texte de synthèse sur l'année hydrologique (faits marquants en termes d'abondance ou de déficit, mention de crues). Le texte de synthèse sera particulièrement soigné sur la période 1947-1952, grâce à la contribution du géographe Charles Péguy avec une analyse météorologique et une interprétation de la variabilité spatio-temporelle des débits. A partir de 1953 le texte sera moins détaillé et centré sur l'hydraulicité des cours d'eau des Alpes, du Massif Central et des Pyrénées. A noter enfin que l'on peut trouver pendant la première décennie (1939-1949 et 1951) un ou deux articles scientifiques par annuaire, dont certains particulièrement intéressants. Le Tableau 2 rappelle les thèmes qui ont pu être traités. Les annuaires fournissent la liste des stations en fonctionnement (de l'ordre de 1000 stations, avec des variations sur la période 1939-1969) et les données journalières-mensuelles-annuelle accompagnées de graphiques sur une sélection de stations. Ces stations dites "fondamentales " (respectivement 68, 82 et 85 en 1939, 1957 et 1969) ont été choisies à partir de critères de qualité, de représentativité spatiale, de longueur de série et d'intérêt opérationnel. Un extrait de l'annuaire 1939 est proposé en Figure 1. En dehors des débits de l'année, d'autres
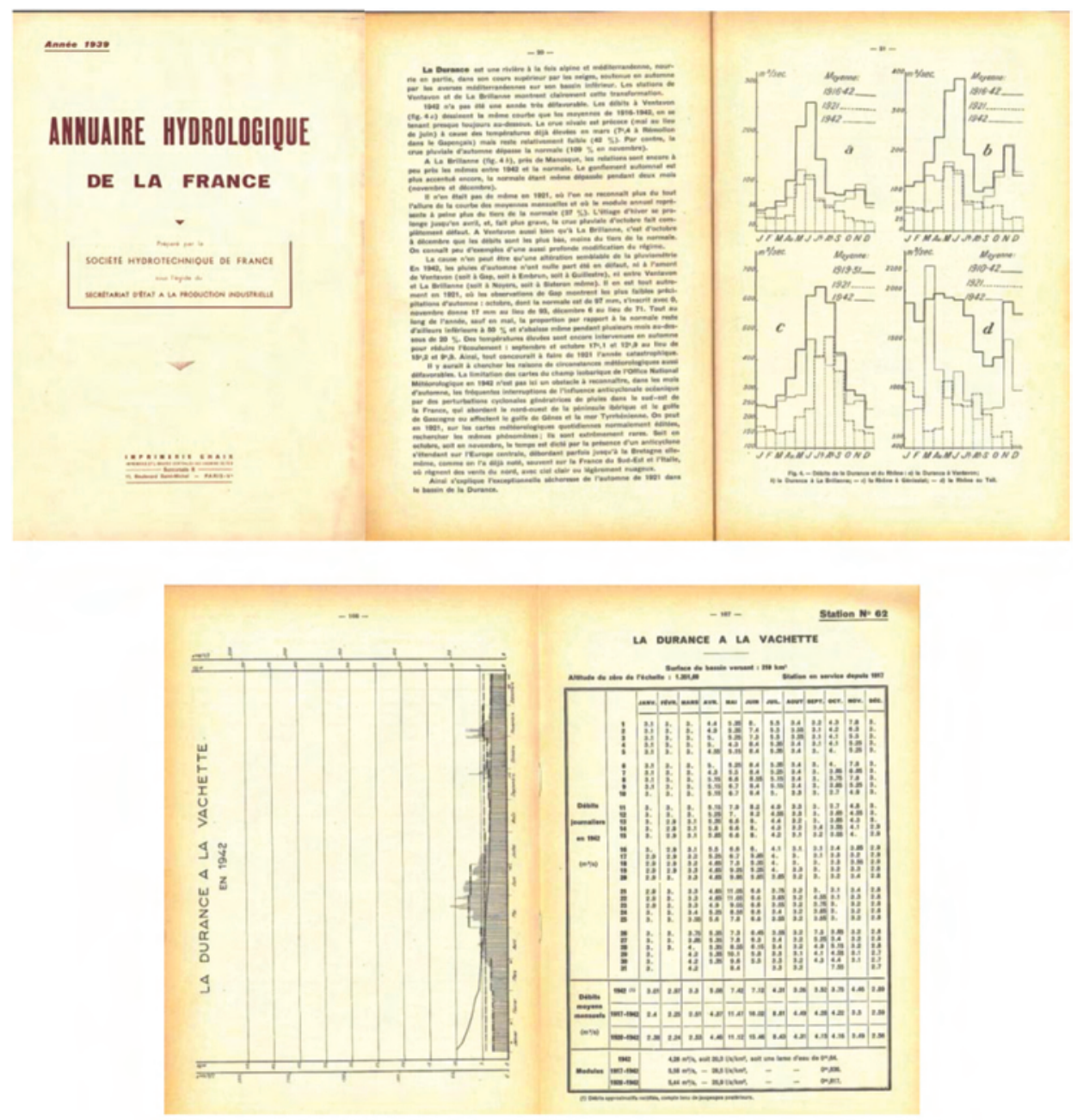

Figure 1 : Extrait de l'annuaire 1939. 
Tableau 1 : Données quantitatives associées aux stations décrites dans les annuaires par période.

\begin{tabular}{|c|c|c|c|c|}
\hline 1939-1947 & 1948-1959 & 1960-1962 & 1963 & 1964-1969 \\
\hline \multicolumn{5}{|c|}{ Surface du bassin versant et altitude (sauf en 1939) } \\
\hline & & & \multicolumn{2}{|c|}{ Latitude et longitude } \\
\hline \multicolumn{5}{|c|}{ Statut de la station (station usine ou non) } \\
\hline \multicolumn{3}{|c|}{ Débits moyens mensuels corrigés de l'année (lorsqu’ils sont disponibles) } & \multicolumn{2}{|c|}{ Moyennes mensuelles mesurées de l'année } \\
\hline \multicolumn{2}{|c|}{$\begin{array}{l}\text { Débits moyens mensuels depuis la mise en service } \\
\text { de la station et jusqu’à l'année considérée }\end{array}$} & \multicolumn{3}{|c|}{$\begin{array}{l}\text { Moyennes mensuelles interannuelles } \\
\text { sur la plus longue période disponible }\end{array}$} \\
\hline \multicolumn{2}{|c|}{$\begin{array}{l}\text { Débits moyens mensuels depuis } 1920 \\
\text { et jusqu'à l'année considérée }\end{array}$} & \multicolumn{3}{|c|}{ Moyennes mensuelles de 1921 à 1960} \\
\hline & & \multicolumn{3}{|c|}{$\begin{array}{l}\text { Maxima et minima journaliers par mois } \\
\text { (valeur en } \mathrm{m}^{3} / \mathrm{s} \text { et année correspondante) }\end{array}$} \\
\hline & & \multicolumn{2}{|c|}{$\begin{array}{l}\text { Valeurs instantanées de débit maximum } \\
\text { par mois de l'année (valeur en m³/s } \\
\text { et jour correspondant) (sauf en 1960) }\end{array}$} & \\
\hline & & \multicolumn{3}{|c|}{ Extrêmes annuels (valeur en m³/s et année correspondante) } \\
\hline
\end{tabular}

informations hydrologiques viennent compléter les tableaux des 365 valeurs journalières. La nature de ces informations a évolué au fil des années (Tableau 1). Chaque annuaire est précédé d'un erratum qui porte sur les annuaires précédents. Il s'agit de corrections à apporter aux métadonnées sur les stations hydrométriques (surface de bassin versant, altitude du zéro de l'échelle limnimétrique, période d'observation) et aussi sur les débits qui ont déjà été publiés (le plus souvent le débit moyen mensuel et parfois le débit moyen journalier).

Deux numéros spéciaux (1950 Alpes et 1951 Massif Central) donnent des informations complémentaires pour combler la lacune sur la période 1925-1938 (hors périodes visées par les Grandes Forces Hydraulique (1911-1924) et par les annuaires de la SHF publiés à partir de 1939). On dispose d'informations en termes de débit moyen mensuel et annuel sur respectivement 23 et 20 stations (Alpes, Massif central) depuis le début du XXe siècle jusque vers 1948 ou 1949. Un numéro spécial analogue sur les Pyrénées était annoncé mais n’a pas été retrouvé dans les différents annuaires disponibles à la Société Hydrotechnique de France.

\section{MÉTHODOLOGIE POUR L'EXTRACTION DES SERIES DES ANNUAIRES}

La numérisation de documents anciens est un enjeu important pour les services d'archives, bibliothèques, historiens, chercheurs... (Coüasnon et al. 2003). De nombreuses techniques de traitement et d'analyse d'images ont été développées ces dernières années, afin de sauvegarder et d'exploiter les informations consignées sous forme papier. Les météorologues (Dandin et al., 2012) se sont engagés dans le sauvetage d'archives et l'extraction de données anciennes. Un exemple est donné par le projet initié par Météo France, les Archives Nationales et la Fondation BNP Paribas et baptisé AAA « Accès aux Archives du climat en dépit de l'Amiante » (http://www.bnpparibas.com/fondation-bnp-paribas/recherche-changement-climatique) qui vise à exhumer les observations des fonds documentaires du service météorologique français, stockés actuellement dans les locaux de Fontainebleau des Archives Nationales.

Le recours à un logiciel d'OCR (Optical Character Recognition ou encore Reconnaissance Optique de Caractères) est incontournable afin d'automatiser au maximum l'extraction. L'OCR est un procédé qui va transformer un fichier image (feuille imprimée, feuillet dactylographié) en un fichier informatique texte exploitable pour des traitements ultérieurs. De nombreux logiciels existent sur le marché. Ils éprouvent des difficultés à extraire des données manuscrites ou issues de supports anciens partiellement endommagés mais sont généralement bien adaptés pour les documents imprimés assez récents (XXe siècle). Nous avons donc choisi d'en utiliser un parmi une sélection de logiciels commerciaux disponibles après des premiers tests effectués sur des feuillets extraits des annuaires. Le travail accompli ici, à la charnière de deux métiers (Gestion électronique des documents et hydrologie) a bénéficié de l'expérience antérieure acquise dans un contexte très différent d'un des coauteurs (Leblois, 2008).

L'obtention des séries présentes dans les annuaires s'est effectuée en trois étapes principales (Figure 2) : (1) la numérisation des fascicules originaux, (2) le traitement des fichiers numérisés par le logiciel d'OCR et (3) la correction et la vérification des données obtenues. Ces trois étapes sont décrites dans les sections suivantes. Notons que nous avons modifié les données publiées lorsqu'un erratum les concernant a été édité.

Afin de ne pas engager inutilement et de manière exhaustive (i.e. sur les 31 années) les étapes (2) et (3) (les plus fastidieuses), nous avons cherché à identifier quelles stations présentes dans les annuaires déjà étaient disponibles dans la banque HYDRO, sur la base des métadonnées disponibles 
Tableau 2 : Liste des 14 articles scientifiques publiés dans les annuaires

\begin{tabular}{|c|c|c|c|}
\hline Annuaire & Auteur & Titre & Commentaire \\
\hline 1939 & Pardé M. & L'hydrologie fluviale française & $\begin{array}{l}\text { Notions générales sur le régime des cours d'eau } \\
\text { (bilan annuel, variations saisonnières, étiages, crues, } \\
\text { transport solide) }\end{array}$ \\
\hline 1940 & Massé P. & $\begin{array}{l}\text { Situation, perspectives et } \\
\text { applications de l'hydrologie } \\
\text { statistique }\end{array}$ & $\begin{array}{l}\text { Etat des lieux encore embryonnaire sur l'hydrologie } \\
\text { statistique (lois de probabilité, oscillations climatiques, } \\
\text { corrélations temporelle et spatiale, prévision à court } \\
\text { et long terme) }\end{array}$ \\
\hline 1940 & Pardé M. & Les crues de 1940 & $\begin{array}{l}\text { Source d'information intéressante sur les crues du } \\
\text { Cher (mai) Garonne (mai et déc.), Isère (sept.) } \\
\text { et surtout Pyrénées orientales (oct.) }\end{array}$ \\
\hline 1941 & Halphen E. & $\begin{array}{l}\text { Etude statistique des débits du Rhin } \\
\text { à Bâle }\end{array}$ & $\begin{array}{l}\text { Analyse statistique (débits moyens annuel et mensuel) } \\
\text { d'une longue série de débits sur le Rhin à Bâle } \\
\text { (1808-1925) }\end{array}$ \\
\hline 1942 & $\begin{array}{l}\text { de Martonne E. } \\
\text { et Bagnens S. }\end{array}$ & Deux années sèches : 1921 et 1942 & $\begin{array}{l}\text { Comparaison des débits mensuels avec les débits } \\
\text { moyens, liens avec le régime pluviométrique, } \\
\text { variabilité spatiale }\end{array}$ \\
\hline 1943 & Waeber $\mathrm{H}$. & \begin{tabular}{|l|}
$\begin{array}{l}\text { Observations sur le régime des } \\
\text { torrents alpestres en haute altitude et } \\
\text { spécialement d'un torrent glaciaire }\end{array}$ \\
\end{tabular} & $\begin{array}{l}\text { Analyse des premières mesures (1940-1943) sur un } \\
\text { petit bassin versant }\left(4 \mathrm{~km}^{2}\right) \text { juste en aval du glacier } \\
\text { de Tré-la-Tête }\end{array}$ \\
\hline 1944 & $\begin{array}{l}\text { Tissier J. et } \\
\text { Zaccagnino E. }\end{array}$ & $\begin{array}{l}\text { Monographie hydrologique de la } \\
\text { Haute Dordogne }\end{array}$ & $\begin{array}{l}\text { Caractéristiques du bassin en termes de relief, } \\
\text { géologie, température, pluie, débit, crues } \\
\text { (8 crues historiques de } \mathbf{1 7 8 3} \text { à 1912) } \\
\end{array}$ \\
\hline 1944 & Léo B. & Mesure et estimation des débits & $\begin{array}{l}\text { Recommandations pour l'hydrométrie (jaugeage, } \\
\text { transfert spatial, estimation à partir des pluies) }\end{array}$ \\
\hline 1945 & Halphen E. & $\begin{array}{l}\text { Un exemple d'application des } \\
\text { méthodes statistiques : le problème } \\
\text { du plan de développement de la } \\
\text { production d'énergie électrique }\end{array}$ & $\begin{array}{l}\text { Exposé pédagogique sur les statistiques appliquées } \\
\text { à l’hydrologie (notions de régions hydrologiques, } \\
\text { simulation de séries, optimisation du productible } \\
\text { électrique) }\end{array}$ \\
\hline 1946 & $\begin{array}{l}\text { Halphen E., } \\
\text { Morlat G. } \\
\text { et Le Cam L. }\end{array}$ & $\begin{array}{l}\text { Sur la valeur industrielle d'une chute } \\
\text { d'eau }\end{array}$ & $\begin{array}{l}\text { Optimisation basée sur une fonction de plusieurs } \\
\text { variables (coût de l'aménagement, bénéfice } \\
\text { pour le soutien d'étiage, économie de charbon) }\end{array}$ \\
\hline 1947 & Serra L. & $\begin{array}{l}\text { Evolution de l'hydrologie : } \\
\text { de la statistique à la physique }\end{array}$ & $\begin{array}{l}\text { Plaidoyer pour introduire des notions physiques en } \\
\text { hydrologie. Analyse de la différence de comportement } \\
\text { de } 3 \text { bassins versants sur l’Aubrac en fonction de } \\
\text { leurs caractéristiques géologiques }\end{array}$ \\
\hline 1948 & Coutagne A. & $\begin{array}{l}\text { Quelques considérations générales } \\
\text { sur la nature, les possibilités } \\
\text { et les modalités des prévisions } \\
\text { en hydrologie fluviale }\end{array}$ & $\begin{array}{l}\text { Possibilités de prévisions hydrologiques à différentes } \\
\text { échéances (régime interannuel, causalité physique - } \\
\text { régime nival, autocorrélation) }\end{array}$ \\
\hline 1949 & Reménieras G. & $\begin{array}{l}\text { L’hydraulique des stations } \\
\text { limnimétriques pour la mesure } \\
\text { du débit des cours d'eau }\end{array}$ & $\begin{array}{l}\text { Article de référence sur les relations hauteur-débit } \\
\text { pour l'hydrométrie (écoulement uniforme, non } \\
\text { uniforme permanent, non permanent). Cas des stations } \\
\text { à deux échelles, courbe d'hystérésis }\end{array}$ \\
\hline 1951 & Gaspard B. & $\begin{array}{l}\text { Note sur la détermination des débits } \\
\text { de la Seine à Paris }\end{array}$ & $\begin{array}{l}\text { Modalités d'estimation du débit de la Seine à Paris et } \\
\text { graphiques de débit (années } 1944 \text { à 1951). Différences } \\
\text { notables avec les débits de la banque HYDRO }\end{array}$ \\
\hline
\end{tabular}

(confrontation des noms, coordonnées, surfaces). Lorsque nous soupçonnions avoir identifié un équivalent dans la banque HYDRO, l'examen des séries a permis de conclure : - lorsque des observations étaient disponibles sur une ou plusieurs année(s) commune(s), nous avons calculé les valeurs absolues des écarts entre les débits journaliers de la station de l'annuaire sur quelques années et ceux de la station de la banque HYDRO qui semblait correspondre.
Nous avons classé les valeurs et considéré les deux stations identiques lorsque le dernier décile est égal à zéro. Il en est de même lorsque le dernier décile est supérieur à zéro mais que la moyenne des différences relatives est inférieure à $5 \%$. Lorsque la moyenne des écarts est supérieure à $5 \%$ mais reste inférieure à $10 \%$, nous avons multiplié les années de comparaison et, finalement, une expertise hydrologique permet de statuer ; 


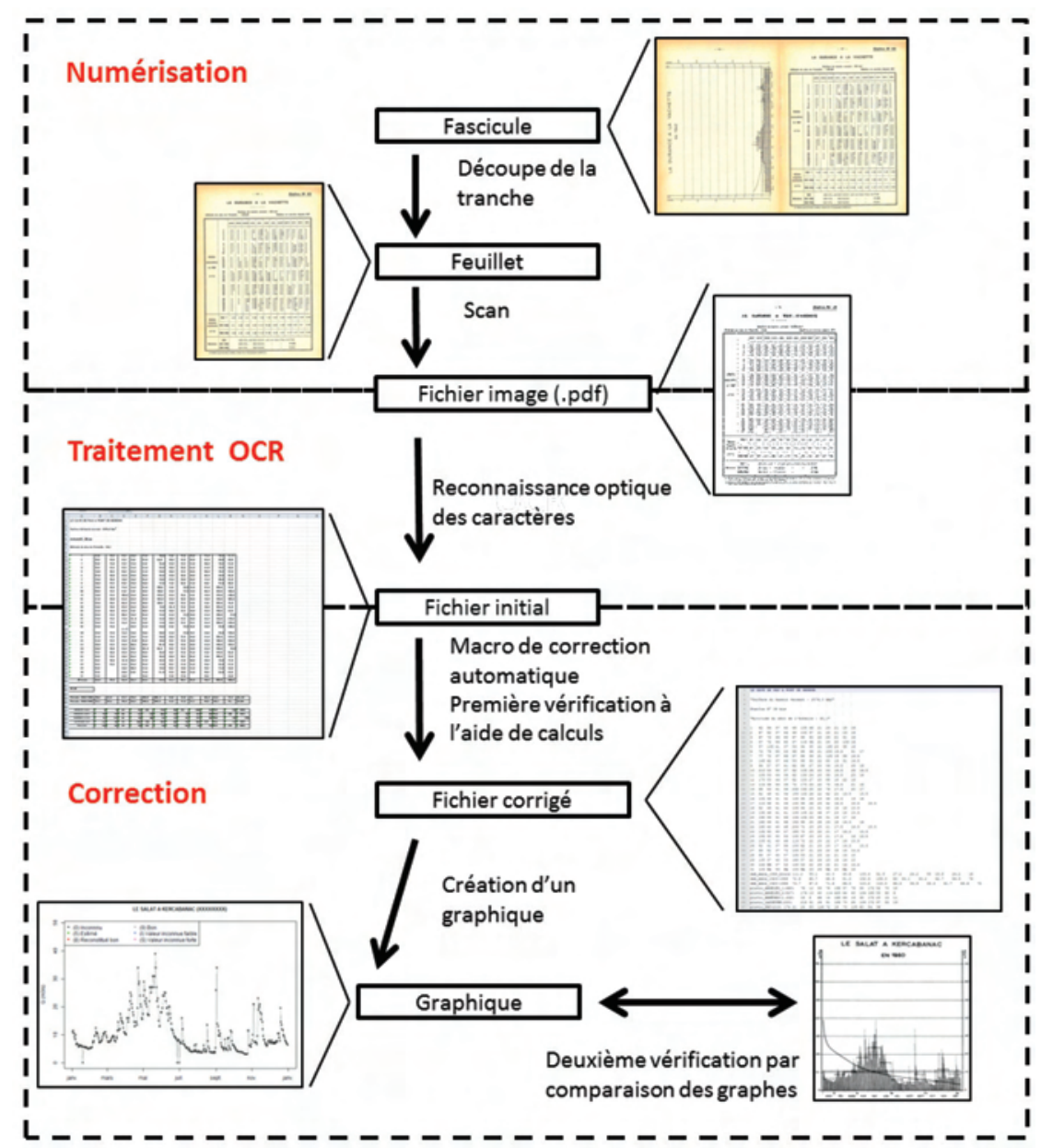

Figure 2 : Schéma récapitulatif des principales étapes du protocole d'extraction des données.

- lorsque la comparaison directe n'était pas possible (pas de chevauchement entre les chroniques des deux stations), des tests de stationnarité (Pettitt, Buishand, Lee et Heghinian, Hubert) ont été appliqués pour nous éclairer sur l’homogénéité de la série concaténée.

\section{III.1. Numérisation des fascicules}

La première phase de la numérisation consiste à scanner les documents, après massicotage des originaux (découpe de la tranche des annuaires). Le choix de la résolution du scan est un critère technique important à définir, déterminant pour la suite des opérations. Compte tenu des documents (état physique des annuaires, polices d'écriture anciennes), la résolution du scan a été fixée à $600 \mathrm{dpi}$, avec détection automatique de couleurs puis binarisation en noir et blanc des pages. Le massicotage et le scan des feuillets ont été externalisés à une société spécialiste de la numérisation. Plus de 9000 pages A4 ont ainsi été scannées et converties au format pdf. Une version dégradée de ces fichiers (300 dpi), plus légère, a été mise en ligne pour consultation et téléchargement sur le site de la SHF (http://www.shf-hydro.org/ annuaires_hydrologiques-73.html).

\section{III.2. Traitement par un logiciel d'OCR}

Une fois les pages numérisées, les images scannées ont été converties en données numériques exploitables.
Si l'ensemble des pages des 31 annuaires hydrologiques a été scanné, seuls les tableaux présentant des données de débits journaliers ont été traités par OCR pour associer à chaque tableau de données (en format pdf) une feuille de calcul de tableur contenant les données numérisées.

L'utilisation d'un modèle de zone permettant d'appliquer à toutes les pages la même structure d'éléments à reconnaitre (textes, tableaux) a facilité le traitement. Cependant un ajustement manuel a été toutefois nécessaire à la marge sur toutes les pages afin que les zones soient bien positionnées (ex. décalage léger entre chaque page, nombre de lignes des tableaux non uniforme...). Il a fallu également corriger les erreurs de structuration des tableaux (lignes à ajouter ou supprimer, cellules fusionnées à séparer...). Nous avons mentionné précédemment l'introduction progressive de nouvelles données dans les tableaux synthétiques (Tableau 1) et les formats des tableaux et les polices d'écriture utilisées ont évolué (Figure 3). Ces transformations ont compliqué l'étape de traitement par OCR, car il a fallu régulièrement réadapter les paramètres du logiciel utilisés (en particulier le modèle de zone).

\section{III.3. Contrôle des fichiers de sortie}

L'agencement des pages, la présence de tableaux plus ou moins bien délimités, l'alternance de texte et de chiffres, les polices anciennes, rendent difficile le travail de reconnaissance de caractères : les erreurs « d'interprétation » 

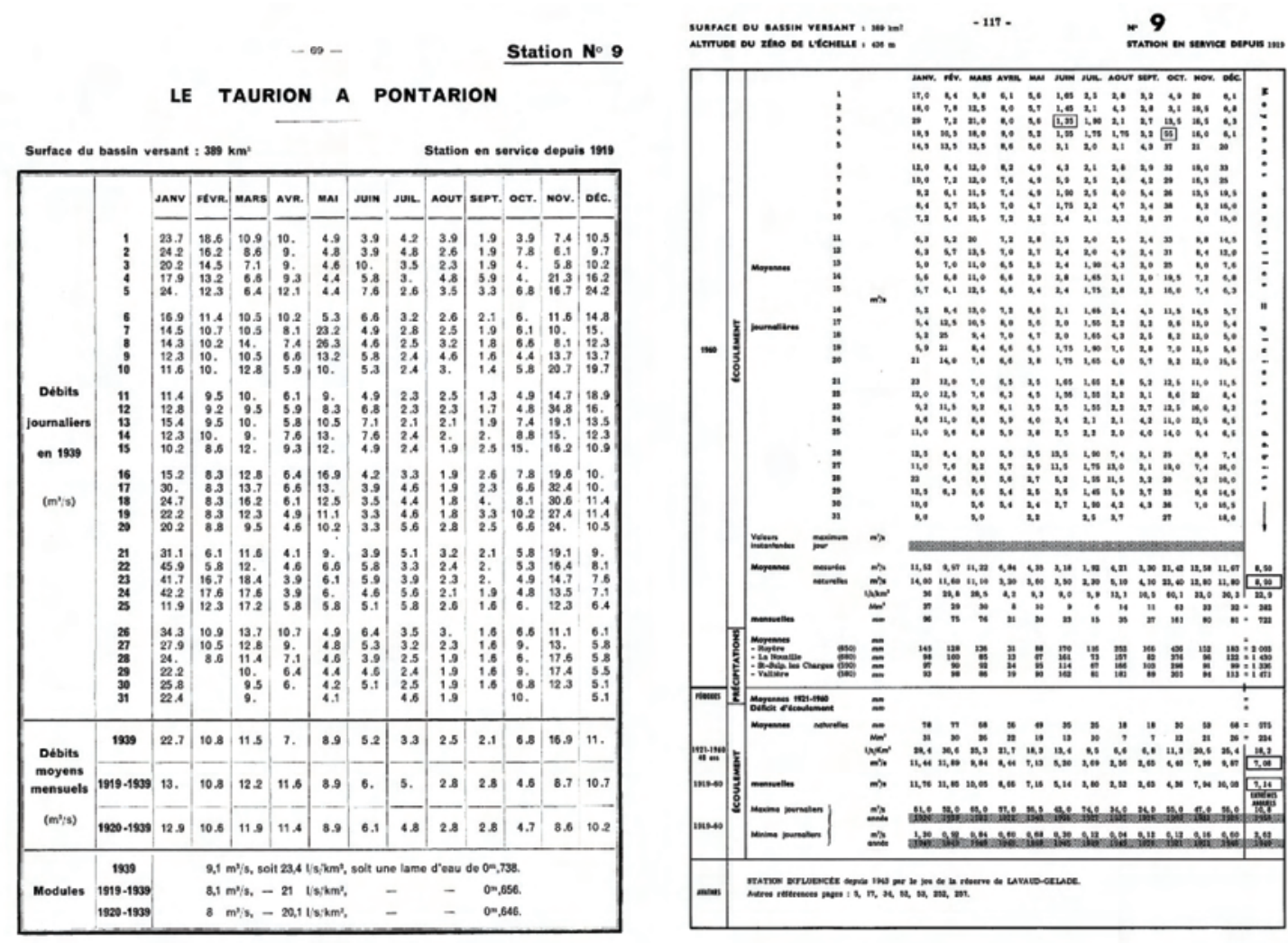

Figure 3 : Exemple de deux formats de tableaux pour les données d'une même station en 1939 (sur la gauche) et en 1960 (sur la droite).

sont fréquentes et les données obtenues ne sont pas assez fiables pour être directement exploitées. Un contrôle des fichiers de sortie est donc essentiel pour s'assurer de la qualité des données numérisées, et le cas échéant les erreurs commises par le logiciel doivent être corrigées. Ce contrôle qualité a été réalisé avec un tableur et a nécessité le codage de différentes macros.

Nous avons rapidement pu identifier des erreurs récurrentes commises par le logiciel d'OCR, ce qui nous a permis de réaliser une procédure semi-automatique d'identification et de correction des erreurs. Parmi ces erreurs types, citons :

— l'ajout de caractères « i », «/ ", «| ", «! », «: " , .. sur des lignes des tableaux lorsque le trait de bordure de la colonne était mal identifié,

— le remplacement d’un « 0 » par un « $\mathrm{O}$ », de « 11 » par un « $U »$, de « 6 » par un « $b » .$.

Ces premières corrections ont été complétées par deux autres phases de vérifications successives : l'une à l'aide de calculs et l'autre à l'aide des graphiques. Les annuaires présentent à la fois les données journalières de débits et les moyennes mensuelles correspondantes. Il est possible de recalculer ces moyennes mensuelles à l'aide des données journalières, et vérifier qu'elles sont similaires à la moyenne mensuelle indiquée dans le document source. Si ce n'est pas le cas, nous pouvons soupçonner une erreur d'identification de la part du logiciel sur des valeurs journalières intervenant dans le calcul en question.

Cette vérification par le calcul présente l'intérêt d’identifier rapidement des erreurs d'OCR liées au remplacement d'un chiffre par une lettre (le calcul de moyenne ne se fait pas). Toutefois, cette vérification n'est pas toujours très efficace en cas de confusion d'un chiffre avec un autre. Une seconde étape de vérification nous a donc paru nécessaire.
En complément, nous avons donc exploité les informations contenues dans les graphiques adjacents aux tableaux de données annuels. Des hydrogrammes ont été élaborés à partir des séries issues de l'étape précédente, ce qui nous a permis de réaliser la seconde étape de vérification de l'OCR. La comparaison visuelle des deux graphiques permet de déceler de nouvelles erreurs.

\section{III.4. Résultats}

Nous avons identifié près de 2300 tableaux synthétiques contenant les 365 ou 366 débits journaliers observés sur un sous-ensemble de stations dans les 31 annuaires hydrologiques. L'examen des redondances " évidentes » avec les données de la banque HYDRO (interrogation en mai 2015) a réduit le nombre de tableaux à environ 1600, répartis sur une centaine de stations hydrométriques.

Ainsi, sur les 120 stations décrites dans les annuaires, trois d'entre elles sont implantées en Suisse (Rhin à Rheinfelden, Doubs à Ocourt, Arve au Pont de Carouge). Les données associées sont mises à disposition pour la période 1993-2015 sur le site de l'Office fédéral de l'environnement OFEV (http://www.hydrodaten.admin.ch/fr). Les données du Rhône à Penay et à Chancy (Aux Ripes) sont bancarisées en France et en Suisse. La Figure 4 fait apparaitre l'ensemble des stations pour lesquelles les débits sont disponibles dans les annuaires. Sans surprise elles sont situées majoritairement dans les secteurs à enjeu énergétique. Finalement, 15 points de mesure semblent correspondre à des stations inédites, non présentes dans la banque HYDRO (Tableau 3 ; Figure 4), et 41 séries apportent des données complémentaires à des stations de la banque HYDRO (Tableau 4 ; Figure 4). Un total 


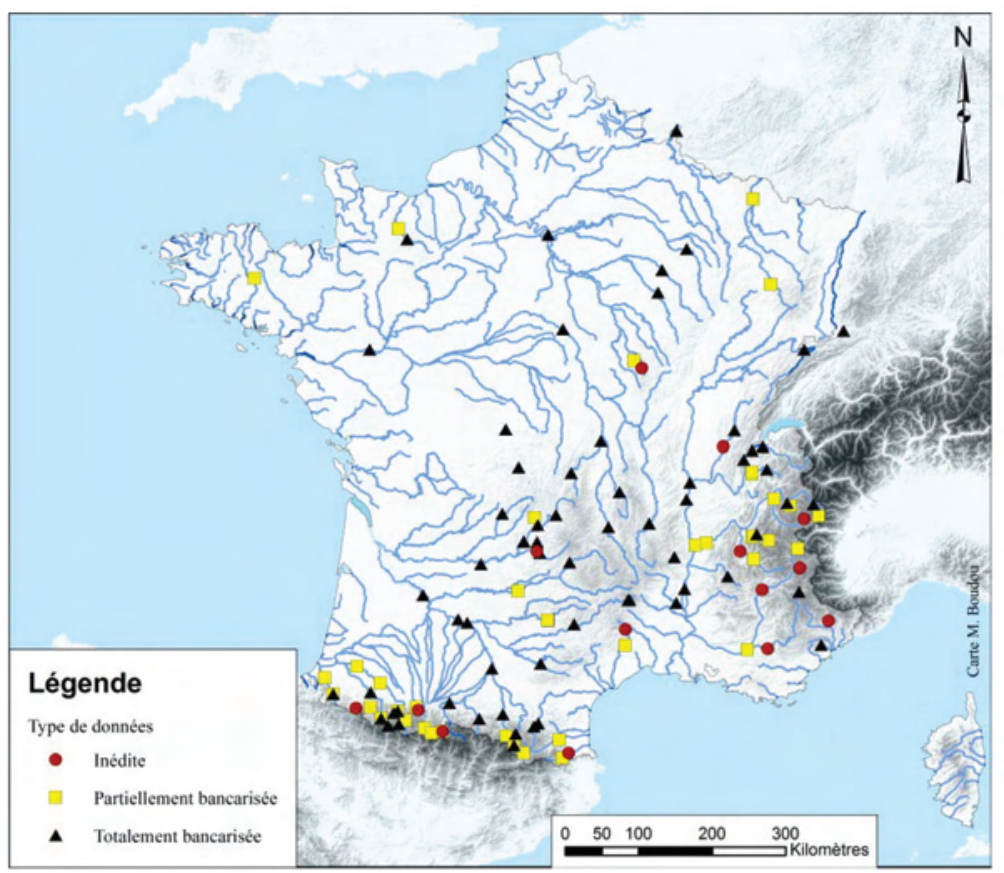

Figure 4 : Localisation et statut des stations présentes dans les annuaires.

Tableau 3 : Descriptif des stations inédites.

\begin{tabular}{|c|c|c|c|c|c|}
\hline $\begin{array}{c}\mathrm{N}^{\circ} \\
\text { (jusqu'en } \\
\text { 1956) }\end{array}$ & $\begin{array}{c}\mathrm{N}^{\circ} \\
\text { (à partir } \\
\text { de 1957) }\end{array}$ & Nom (annuaire) & $\begin{array}{c}\text { Surface } \\
\left(\mathbf{k m}^{2}\right)\end{array}$ & $\begin{array}{l}\text { Date de mise } \\
\text { en service }\end{array}$ & $\begin{array}{l}\text { Chronique } \\
\text { disponible }\end{array}$ \\
\hline 3 & & LA CURE AUX SETTONS & 50 & 1926 & 1939 \\
\hline 16 & & LA CERE A MONTVERT (Usine de Lamativie) & 764 & 1909 & $1939-1948$ \\
\hline 24 & & LA PIQUE A LA PIQUE SUPÉRIEURE & $50.5 / 58.6$ & 1931 & $1939-1947$ \\
\hline 33 & & L'ADOUR A ARTHEIL & 160.6 & 1912 & $1939-1941$ \\
\hline 36bis & $36-1$ & $\begin{array}{l}\text { LE GAVE DE SAINTE ENGRACE } \\
\text { A LICQ-ATHEREY }\end{array}$ & 61 & & $1956-1969$ \\
\hline \multirow[t]{2}{*}{41} & & LE TECH A CANTAÏRÉ & 393 & 1920 & $1939-1940$ \\
\hline & $42-3$ & L'HERAULT A LA VALETTE & 109 & & $1957-1958$ \\
\hline 44 & 44 & L’AIN A CIZE-BOLOZON & 2560 & 1923 & $1939-1969$ \\
\hline 56 & & L’ARC A TERMIGNON & 359 & 1924 & 1939 \\
\hline 58 & & LE DRAC A AVIGNONET & $1968 / 2006.7$ & 1904 & $1939-1954$ \\
\hline 63 & 63 & LA DURANCE A VENTAVON & 4215 & 1912 & 1939-1969 \\
\hline 65 & & LE GUIL A PONT-LA-PIERRE & 475 & 1914 & 1939-1948; 1952 \\
\hline 65bis & $65-1$ & LE GUIL A LA MAISON DU ROY & 580 & & $\begin{array}{c}1953-1958 ; \\
1960-1969\end{array}$ \\
\hline 67 & 67 & LE VERDON A QUINSON & 1661 & 1906 & 1939-1969 \\
\hline 68 & 68 & LA TINÉE A BANCAIRON & $532 / 450$ & 1929 & 1939-1969 \\
\hline
\end{tabular}

de 577 années stations inédites ont été exhumées (209 sont associées aux stations inédites).

\section{III.5. Problèmes rencontrés}

Le travail d'extraction des données des annuaires hydrologiques publiés par la SHF a soulevé un certain nombre de difficultés, malgré l'existence d'outils d’OCR performants.
Certaines d'entre elles sont décrites ici, afin de donner une idée aux lecteurs des complications qu'ils pourraient rencontrer lors de l'extraction de données anciennes. La qualité de l'impression est un paramètre déterminant pour une bonne reconnaissance par OCR. Certains tableaux, rédigés à la machine à écrire, ne présentaient pas toujours une qualité suffisante pour nous permettre d'être sûrs de ne pas faire d'erreur de lecture : décalage de lignes, caractères effacés... 


\begin{tabular}{|c|c|c|c|c|c|c|c|c|c|c|c|c|c|c|c|c|c|c|}
\hline 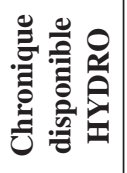 & हू & & 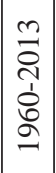 & $\mid \begin{array}{l}\vec{\infty} \\
o \\
\sigma \\
\hat{b} \\
\sigma \\
\sigma \\
-\end{array}$ & 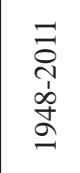 & $\begin{array}{l}0 \\
2 \\
\sigma \\
T \\
\hat{1} \\
\hat{\sigma} \\
-\end{array}$ & $\begin{array}{l}\vec{\nabla} \\
\stackrel{1}{\Delta} \\
\vec{d}\end{array}$ & 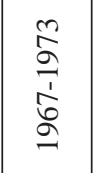 & 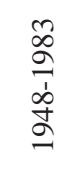 & 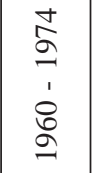 & 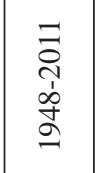 & 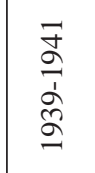 & 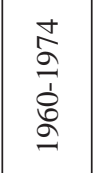 & 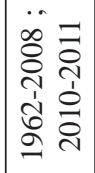 & 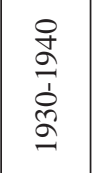 & 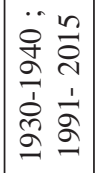 & 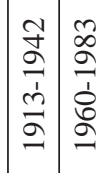 & 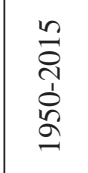 \\
\hline 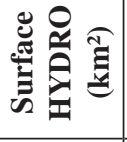 & I & E & $\begin{array}{l}\infty \\
\infty \\
⿱ ㇒ ⿲ 丶 丶 ㇒\end{array}$ & $\infty$ & రิ & $\mid$ & ิㅡㅁ & 占 & $\begin{array}{l}\infty \\
\text { ஸ̂. }\end{array}$ & $\vec{\infty}$ & ஜ் & $\widehat{0}$ & $\stackrel{1}{1}$ & 兽 & 웅 & 号 & 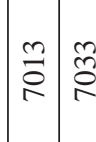 & ลิ \\
\hline 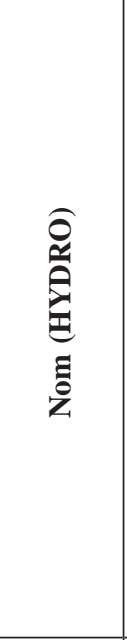 & 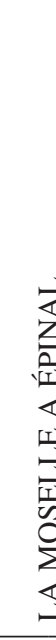 & & 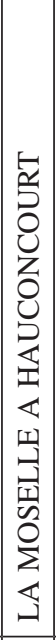 & 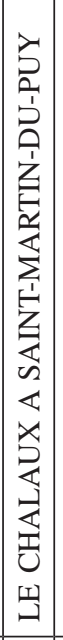 & 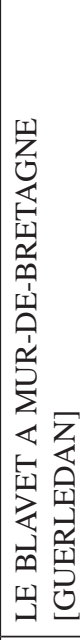 & 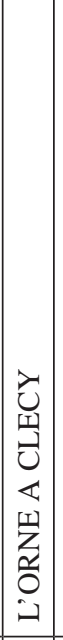 & 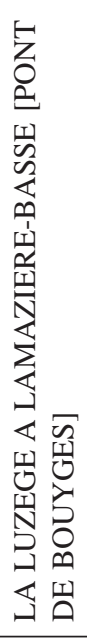 & 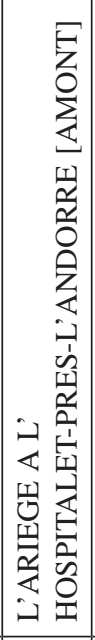 & 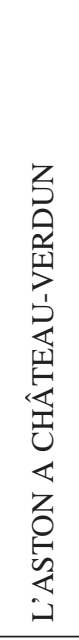 & 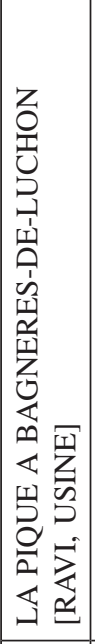 & 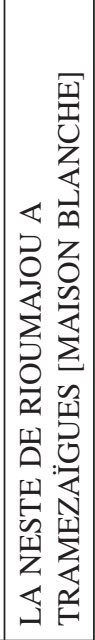 & 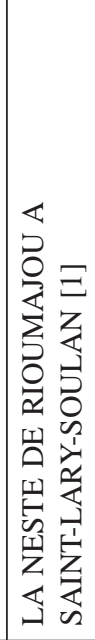 & 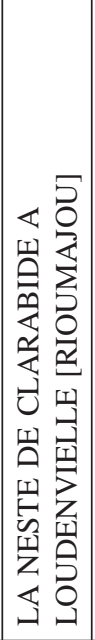 & 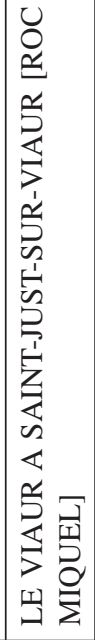 & 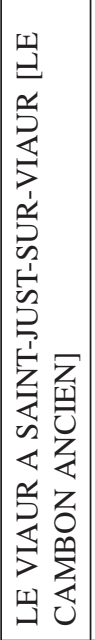 & 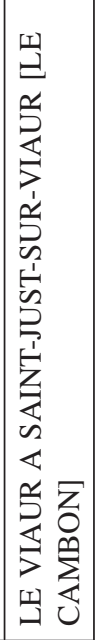 & 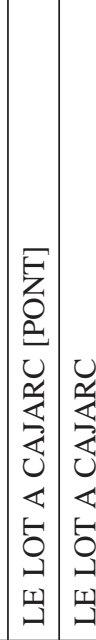 & 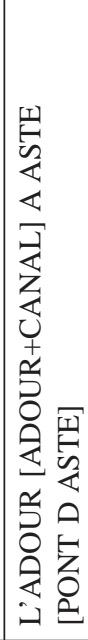 \\
\hline 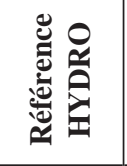 & & & \begin{tabular}{|l|}
0 \\
0 \\
0 \\
0 \\
0 \\
$\sigma$ \\
$\sigma$ \\
\multirow{2}{4}{} \\
\end{tabular} & \begin{tabular}{|c|}
0 \\
$\overrightarrow{1}$ \\
$\bar{m}$ \\
$\vec{M}$ \\
$\vec{I}$ \\
\end{tabular} & 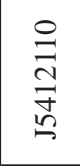 & $\begin{array}{l}0 \\
0 \\
0 \\
0 \\
0 \\
m \\
m\end{array}$ & 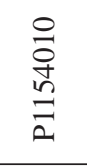 & 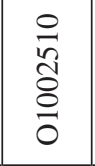 & 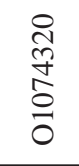 & 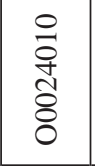 & 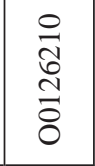 & $\begin{array}{l}\text { స్ } \\
\text { ڤ్ } \\
\text { రి }\end{array}$ & 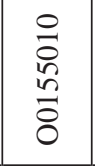 & 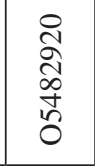 & $\begin{array}{l}\text { ద్ } \\
\text { నे } \\
\text { Oे } \\
\text { Lे } \\
0\end{array}$ & $\begin{array}{l}\text { Oे } \\
\text { Oे } \\
\infty \\
0 \\
0 \\
0\end{array}$ & 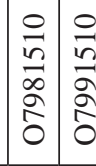 & 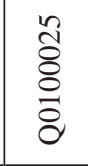 \\
\hline 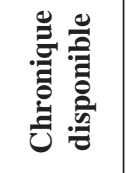 & $\begin{array}{l}\vec{\sigma} \\
\sigma \\
\vec{\sigma} \\
\tilde{\sigma} \\
\sigma\end{array}$ & 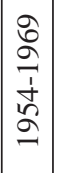 & 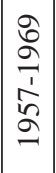 & 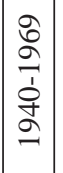 & 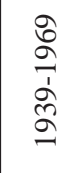 & 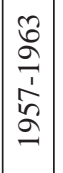 & $\begin{array}{l}8 \\
8 \\
\frac{9}{1} \\
\frac{1}{9} \\
\stackrel{9}{7}\end{array}$ & 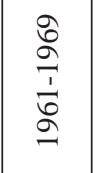 & 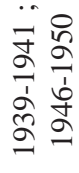 & $\begin{array}{l}\not{g} \\
\frac{1}{1} \\
\text { Щू. }\end{array}$ & & & 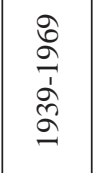 & & 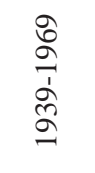 & & 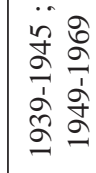 & 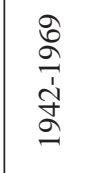 \\
\hline 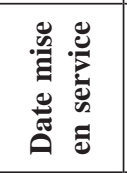 & $\mid$\begin{tabular}{|l}
$\vec{D}$ \\
$\stackrel{\sim}{\sim}$
\end{tabular} & 岕 & 总 & 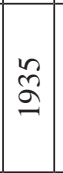 & สี & 峉 & 鸪 & $\begin{array}{l}\overrightarrow{8} \\
\vec{\sigma}\end{array}$ & $\vec{\sigma}$ & $\begin{array}{l}\vec{N} \\
\vec{J}\end{array}$ & & 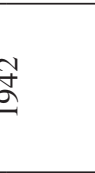 & $\stackrel{\mathscr{N}}{\sigma}$ & & $\overrightarrow{\widetilde{\sigma}}$ & & $\stackrel{m}{g}$ & $\vec{\sigma}$ \\
\hline 芯 & 号 & 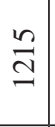 & 守 & \begin{tabular}{|c|}
8 \\
$\stackrel{1}{1}$ \\
$\infty$ \\
$o$
\end{tabular} & ర్రి & $\mid$\begin{tabular}{l}
0 \\
\multirow{2}{*}{} \\
|
\end{tabular} & ญิ & 夺 & 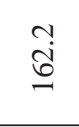 & $\vec{\infty}$ & & 8 & $\stackrel{\bullet}{-1}$ & & 吕 & & 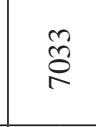 & Nิ \\
\hline 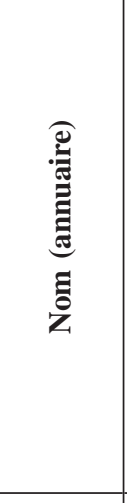 & 4 & 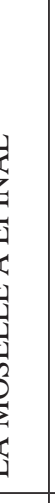 & 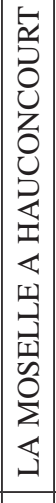 & 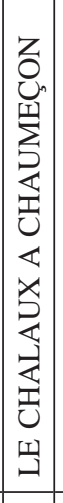 & 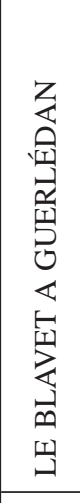 & 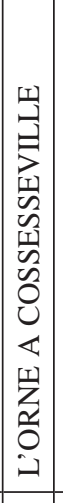 & 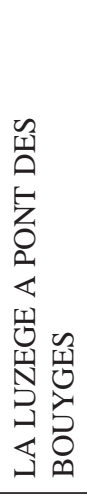 & 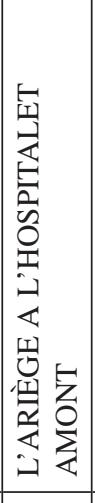 & 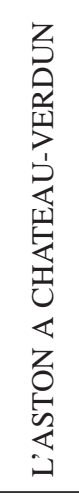 & 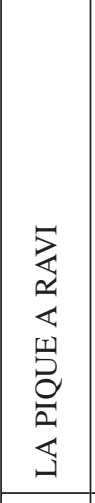 & 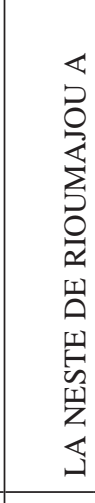 & . & 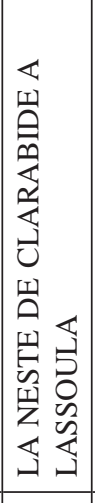 & & 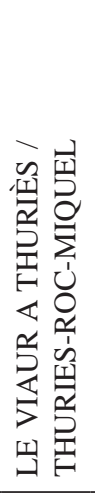 & & 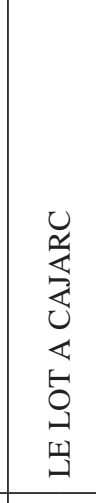 & 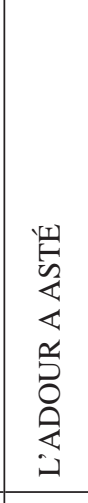 \\
\hline 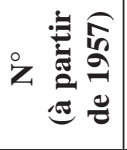 & & $\vec{\sim}$ & $\hat{\sim}$ & $\infty$ & $\nabla$ & $\vec{t}$ & $\stackrel{+}{\sim}$ & $\frac{T}{\Delta}$ & & $\stackrel{d}{\sim}$ & & & $\stackrel{\sim}{\sim}$ & & ని & & $\vec{m}$ & लें \\
\hline 号蓄 & $N$ & तิ & & $m$ & 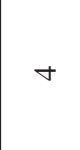 & & 守 & & $\ddot{\vartheta}$ & A & & N & $\stackrel{\sim}{\sim}$ & & $\stackrel{N}{ }$ & & $\vec{m}$ & m \\
\hline
\end{tabular}




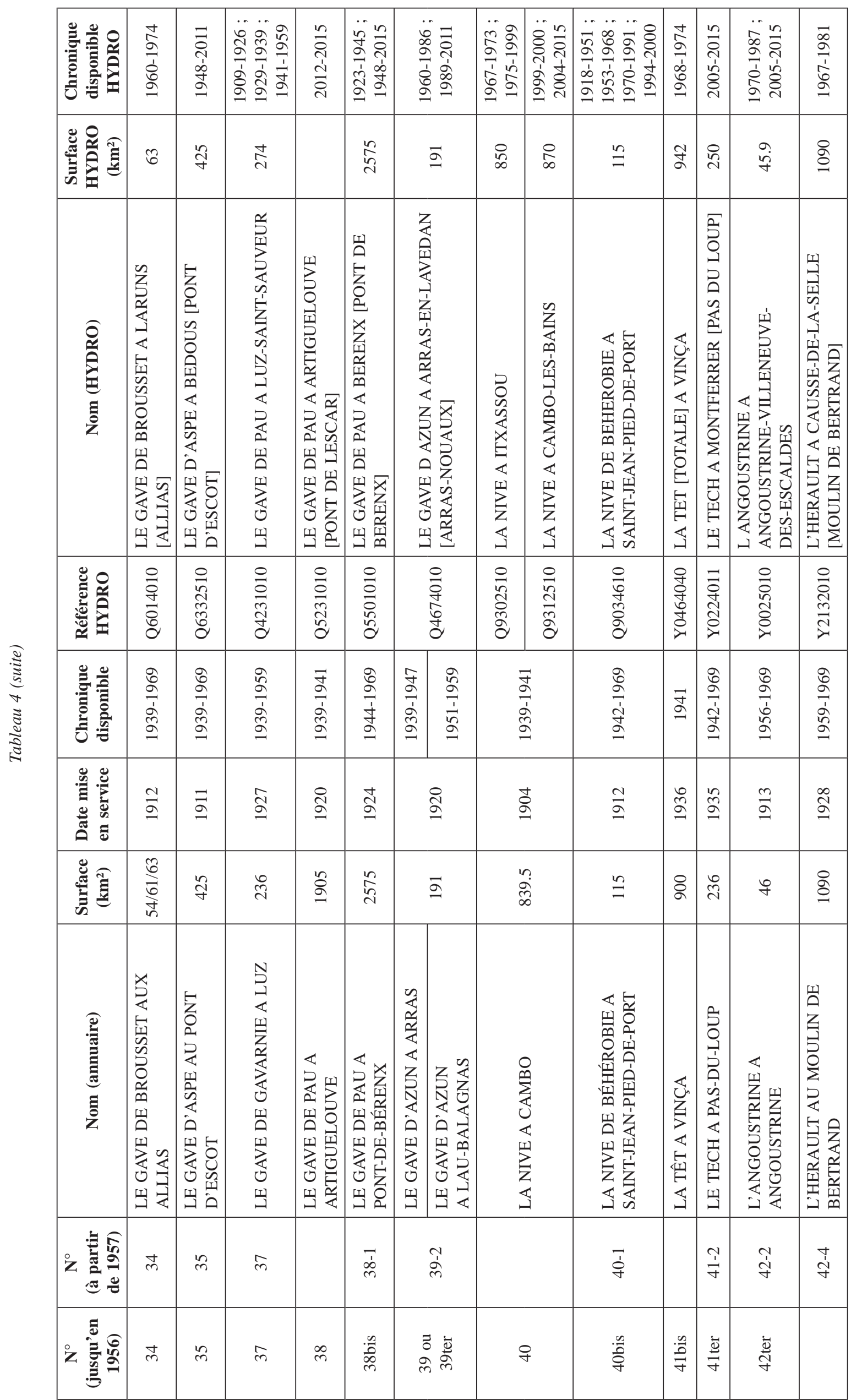




\begin{tabular}{|c|c|c|c|c|c|c|c|c|c|c|c|c|c|c|c|}
\hline 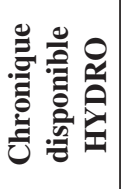 & 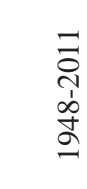 & & 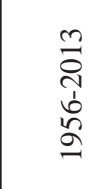 & & 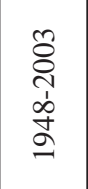 & 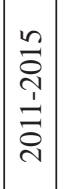 & 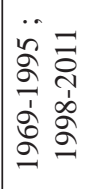 & 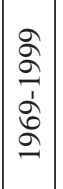 & 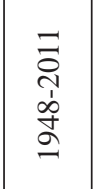 & \multicolumn{2}{|c|}{$\begin{array}{l}\vec{I} \\
\text { गे } \\
\text { मे } \\
\text { g }\end{array}$} & 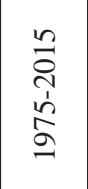 & 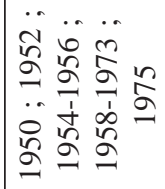 & & 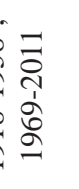 \\
\hline 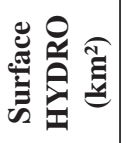 & 品 & & $\begin{array}{l}\stackrel{8}{\infty} \\
= \\
=\end{array}$ & & ల్ల & $\left|\begin{array}{l}0 \\
0 \\
J\end{array}\right|$ & $\begin{array}{l}\stackrel{+}{\dot{b}} \\
\text { 它 }\end{array}$ & ৪ & సิ & \multicolumn{2}{|c|}{$\stackrel{\square}{\circ}$} & 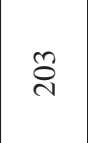 & $\stackrel{\circ}{\vec{N}}$ & & 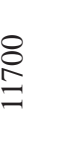 \\
\hline 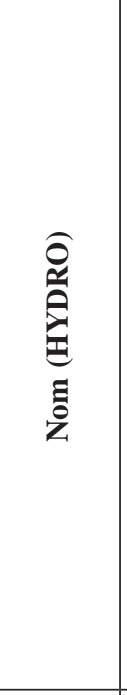 & \multicolumn{2}{|c|}{ 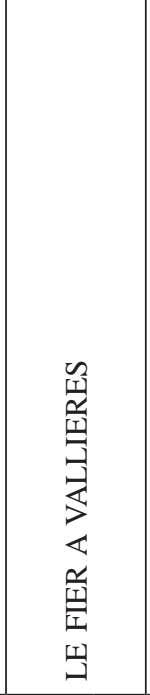 } & 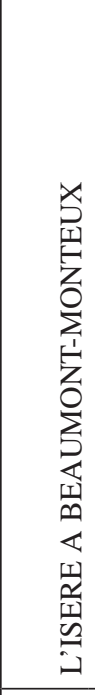 & & 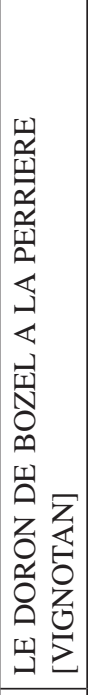 & 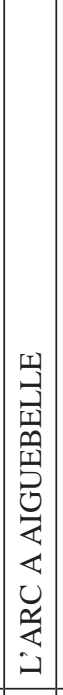 & 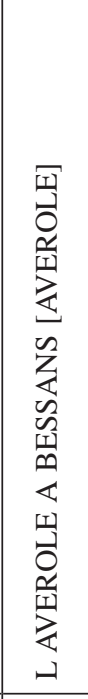 & 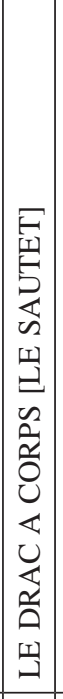 & 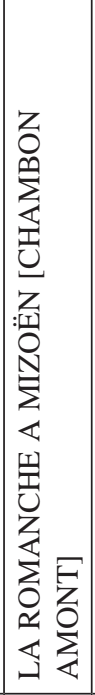 & \multicolumn{2}{|c|}{ 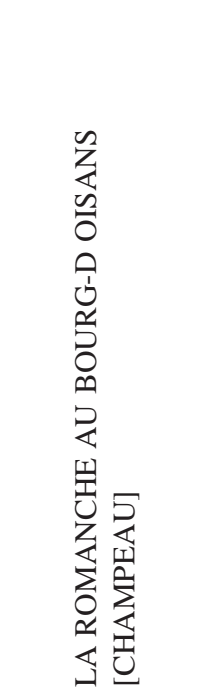 } & 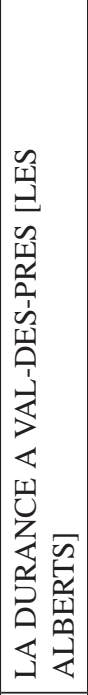 & 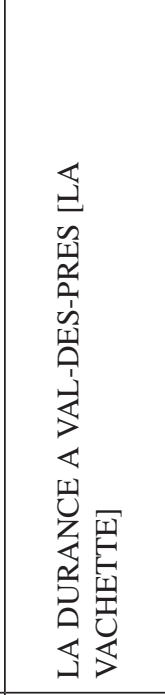 & & 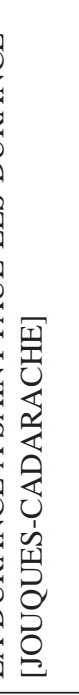 \\
\hline 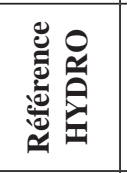 & \multicolumn{2}{|c|}{ 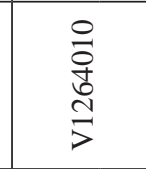 } & \multicolumn{2}{|c|}{$\begin{array}{l}\circ \\
\stackrel{1}{0} \\
\text { ơ } \\
\text { dn } \\
3 \\
3\end{array}$} & 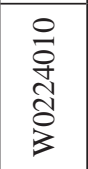 & \begin{tabular}{|l|}
0 \\
0 \\
0 \\
+ \\
0 \\
0 \\
3 \\
\end{tabular} & $\begin{array}{l}0 \\
0 \\
0 \\
0 \\
0 \\
3\end{array}$ & 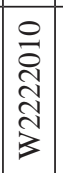 & \begin{tabular}{|l|}
0 \\
0 \\
0 \\
\multirow{2}{1}{} \\
3 \\
3
\end{tabular} & \multicolumn{2}{|c|}{ 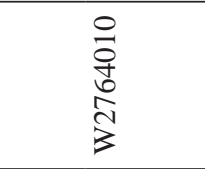 } & 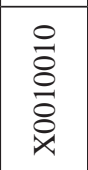 & 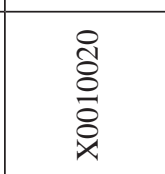 & \multicolumn{2}{|c|}{ 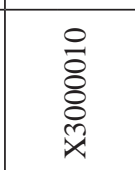 } \\
\hline 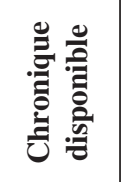 & 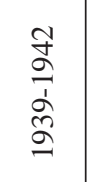 & 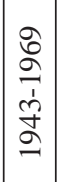 & 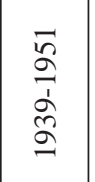 & 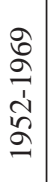 & 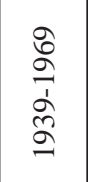 & 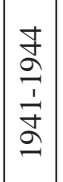 & 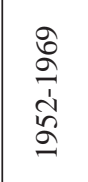 & 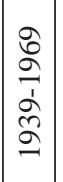 & 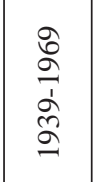 & 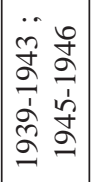 & 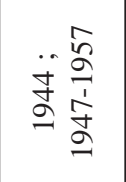 & & 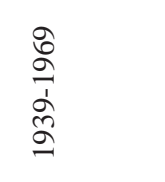 & 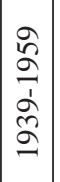 & 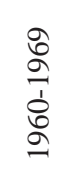 \\
\hline 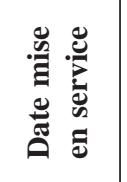 & न्न & $\begin{array}{l}g \\
\mathcal{g} \\
\mathcal{g}\end{array}$ & 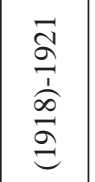 & $\underset{\widetilde{N}}{\mathscr{\sigma}}$ & 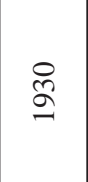 & 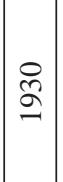 & 吕 & 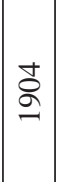 & ల్ల్లి & $\begin{array}{l}\mathscr{b} \\
\sigma \\
\overrightarrow{0} \\
\sigma \\
\sigma \\
\sigma\end{array}$ & 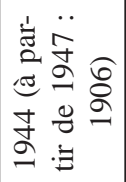 & & 馬 & $\begin{array}{l}\stackrel{+}{0} \\
\stackrel{\sigma}{2}\end{array}$ & 芯 \\
\hline 总 & 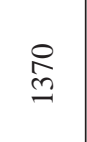 & 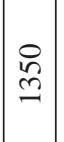 & $\begin{array}{l}8 \\
\text { 足 } \\
=\end{array}$ & $\begin{array}{l}\stackrel{\hat{N}}{\tilde{m}} \\
=\end{array}$ & $\begin{array}{l}\vec{c} \\
\text { ch } \\
\text { o } \\
\text { m }\end{array}$ & $\mid \begin{array}{c}\infty \\
\widetilde{J} \\
\sigma\end{array}$ & $\mathscr{f}$ & 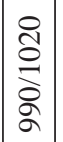 & $\stackrel{\mathcal{N}}{ }$ & કે & $\stackrel{\text { }}{\stackrel{\sigma}{\sigma}}$ & & $\stackrel{ }{\sim}$ & $\begin{array}{l}\stackrel{\curvearrowright}{2} \\
\underset{\sigma}{\sigma}\end{array}$ & \begin{tabular}{l}
$\stackrel{్}{ }$ \\
\multirow{్}{*}{}
\end{tabular} \\
\hline 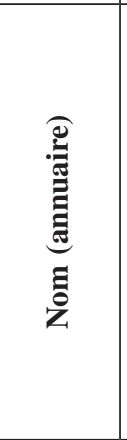 & 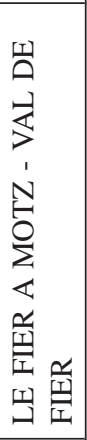 & 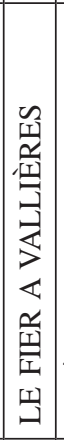 & 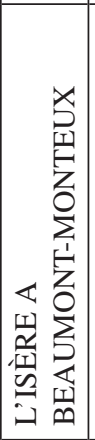 & 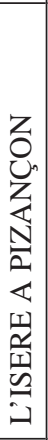 & 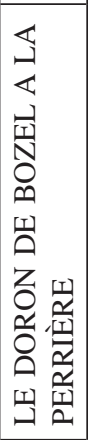 & 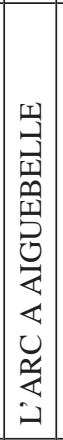 & 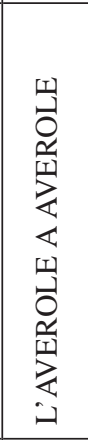 & 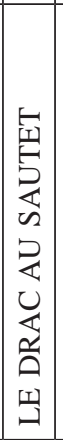 & 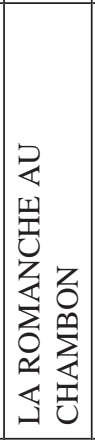 & 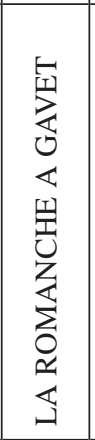 & 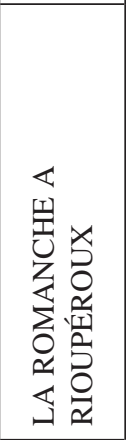 & & 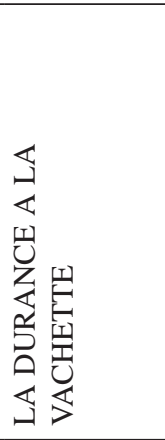 & 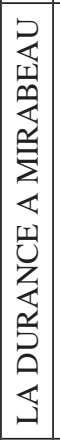 & 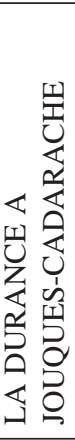 \\
\hline 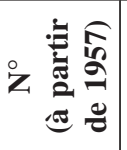 & & $\overrightarrow{\grave{N}}$ & & $\vec{t}$ & 员 & & 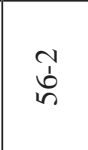 & in & 욤 & & 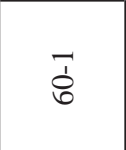 & & $\widetilde{\sigma}$ & 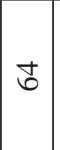 & $\vec{f}$ \\
\hline 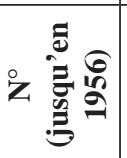 & กิ & $\begin{array}{l}\stackrel{0}{\hat{\lambda}} \\
\tilde{n}\end{array}$ & ષ્ & 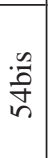 & 놈 & $\mid \begin{array}{l}: 0 \\
0 \\
0 \\
n\end{array}$ & 离 & is & 욤 & 8 & 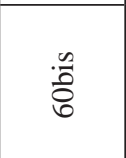 & & $\widetilde{్}$ & 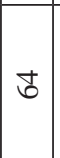 & \\
\hline
\end{tabular}


L'ancienneté des documents que l'on souhaite récupérer peut, en elle-même, poser problème en raison de la difficulté de vérifier des informations datant de plusieurs dizaines d'années. Il faut accepter qu'il existe une part de doute dans les données récupérées, en raison de l'impossibilité de vérifier d'éventuelles erreurs. Nous avons pu constater des incohérences entre les tableaux de données et les graphiques correspondant, et entre les moyennes indiquées dans les tableaux et les moyennes recalculées. Il est impossible de connaître la raison de ces incohérences (erreur de calcul, faute de frappe, correction ultérieure d'une donnée...). Quand les erreurs étaient évidentes, elles ont été corrigées. Le temps nécessaire pour la récupération des données par annuaire a été très variable : il a évolué entre 4 heures pour la décennie 1940 et 10 heures pour la décennie 1960. Cette augmentation est pour partie liée au nombre de données à extraire, et donc au nombre de données à vérifier. Par exemple un tableau présentant les valeurs de débit mensuel maximum et minimum sur une période longue requiert plus de temps pour la vérification, car il contient un grand nombre de données non vérifiables par le calcul, et des chiffres à saisir manuellement (les années, inscrites ici sur un fond grisé, n’ont pas été bien reconnues par l'OCR). La complexité croissante des tableaux a également allongé considérablement le temps d'ajustement des modèles de zone.

Les annuaires présentent des données de débits journaliers sur 120 stations hydrologiques, pour la période 1939-1969. Durant cette période, les stations hydrologiques ont évolué : certaines stations ont été déplacées, remplacées par d'autres, ont changé de nom car la dénomination n'était plus appropriée... Les annuaires ne sont pas toujours très explicites sur ces évolutions, et des erreurs se glissent régulièrement au milieu d'informations exactes. Il y a ainsi beaucoup d'incohérences temporelles sur les numéros et noms de station, les valeurs d'altitude, de surface du bassin versant, les dates de mise en service, les commentaires... Il est donc parfois difficile de savoir si des chroniques de débits journaliers mesurés deux années consécutives l'ont été à la même station, ou si la station était différente la seconde année. Le problème de fiabilité des informations prend une autre dimension quand il s'agit de confronter deux sources de données. Nous avons cherché à identifier des stations identiques entre les annuaires et la banque HYDRO. Outre l'évolution des dénominations des stations et les incohérences des informations présentées dans les annuaires, des corrections réalisées ultérieurement sur les données de la banque HYDRO (modification des courbes de tarage par exemple), ont pu creuser l'écart entre les valeurs extraites des deux sources, pourtant initialement identiques. Pour ces deux raisons, il persiste un doute sur la correspondance réelle entre certaines stations décrites dans les annuaires et les stations décrites dans la banque HYDRO.

\section{CONCLUSION}

Les séries hydro-météorologiques anciennes ont une valeur manifeste pour la connaissance générale du cycle de l'eau et de manière pragmatique pour la mise en place de moyens de prévention contre les événements extrêmes (peu échantillonnés dans les séries courtes). Elles permettent de mieux apprécier la variabilité temporelle des débits dans une perspective de changement climatique. Elles sont le support de validation direct ou indirect de travaux de reconstitution de séries climatiques en France (Caillouet et al.,
2015 ; Kuentz et al., 2015 ; Minvielle et al., 2015). La prise en compte de ces séries anciennes offre enfin une source d'information non négligeable pour parfaire la connaissance d'évènements exceptionnels passés, souvent peu connus et dont l'occurrence future demeure un enjeu de taille pour les gestionnaires du risque (Boudou, 2015).

La communauté des météorologues s'est déjà mobilisée pour identifier des sources potentielles de données, numériser les archives et sauvegarder les observations sur un support numérique. Nous avons souhaité engager une démarche similaire en se focalisant sur les annuaires hydrologiques publiés par la Société Hydrotechnique de France, qui avaient l'intérêt de centraliser une information multi-sites sur une période suffisamment ancienne pour espérer identifier des données inédites.

Les annuaires de 1939 à 1969 ont été numérisés et sont désormais accessibles (http://www.shf-hydro.org/annuaires_ hydrologiques-73.html). Le contenu de ces documents a été détaillé ici. Outre les données pour chaque année, des contributions libres de différents auteurs apportent des éléments d'expertise utile pour les hydrologues. La récupération des séries via un procédé d'OCR est rappelée. Il requiert une phase de contrôle qui peut être longue et fastidieuse selon la complexité du support de base numérisé. Elle a porté ses fruits : nous avons pu exhumer un ensemble de 56 stations vraisemblablement inédites ou apportant des compléments possibles de chroniques pour des stations partiellement bancarisées (un équivalent d'environ 58 années stations), soit près de $50 \%$ des stations mentionnées dans les annuaires.

Il reste à définir une stratégie de mise à disposition de ces données. Elles ne sont pas forcément directement exploitables. Un travail de qualification des données pour une exploitation optimale devra être engagée en s'appuyant sur les commentaires relevés sur les annuaires (sauvegardés également) et au regard des techniques de mesure employées en s'inspirant des travaux de Kuentz (2013). Ce travail de sauvegarde permettra dans un premier temps de valider un modèle hydrologique élaboré sur le Rhône (projet " Modélisation Hydrologique Distribuée du Rhône ») sous réserve de réajustement climatique sur des stations « inédites » ou qui présentent un régime influencé sur la période récente.

\section{REMERCIEMENTS}

Cette action d'extraction des séries a été réalisée dans le cadre du projet de recherche "Modélisation Hydrologique Distribuée du Rhône ", qui vise à déployer un modèle distribué à base physique sur le Rhône et ses affluents majeurs (partie française). Ce projet est soutenu financièrement par l'Agence de l'Eau Rhône-Méditerranée-Corse, la Compagnie Nationale du Rhône et le fond européen FEDER (action Présage 45537).

\section{REFERENCES}

Boudou M. (2015) - Caractérisation de neufs évènements remarquables d'inondations en France au $\mathrm{xx}^{\mathrm{e}}$ siècle : contribution à la valorisation de l'information historique pour la gestion prospective des risques. Thèse, Montpellier 3 en cours.

Caillouet L., Vidal J.-P., Sauquet E., Graff B. (2015) Probabilistic precipitation and temperature downscaling of 
the Twentieth Century Reanalysis over France. Climate of the Past, submitted

Cote E.-F. (1908) — Le service d'étude des grandes forces hydrauliques. La Houille Blanche. 12 : 284-287

CoÜASnON B., Dalbera J.-P., EMPtoz H. (2003) - Numérisation et patrimoine. Document numérique. 7(3-4) : 188 pages

Dandin P., Aressy P., Deaux N., Dubuisson B., Fleuter G., Gibelin A.-L., Jourdain S., Laval L., Menassere S., Roucaute E. \& WieczoreK A.-M. (2012) - Data rescue: a necessary look at climate. In Climate ExChange, WMO, (http:// digital.onwindows.com/climate-exchange/). 268-271

Giuntoli I., Renard B., Vidal J.-P. Bard A. (2013) — Low flows in France and their relationship to large-scale climate indices. Journal of Hydrology. 482 : 105-118

Kuentz A. (2013) - 100 ans de variabilité climatique observée et reconstituée sur le bassin de la Durance. Thèse, AgroParisTech, Paris. 373 pages

Kuentz A., Mathevet T., Gailhard J., Hingray B. (2015) Building long-term and high spatio-temporal resolution precipitation and air temperature reanalyses by mixing local observations and global atmospheric reanalyses: the ANATEM method. Hydrol. Earth Syst. Sci. Discuss. 12 : 311-361

Lang M., Arnaud P., Carreau J., Deaux N., Dezileau L. Garavaglia F., Latapie A., Neppel L., Paquet E., Renard B., Soubeyroux J.M., Terrier B., Veysseire J.M., Aubert Y., Auffray A., Borchi F., Bernardara P., Carre J.C., Chambon D., Cipriani T., Delgado J.L., Doumenc H., Fantin R., Jourdain S., Kochanek K., Paquier A., Sauquet E. Tramblay Y. (2014) - Résultats du projet ExtraFlo (ANR 2009-2013) sur l'estimation des pluies et crues extrêmes. La Houille Blanche. 2(5) : 13

Leblois E. (2008) - Extraction de lexique bilingue à partir d'un corpus de traduction : une stratégie par écrémage. Actes des $9^{\text {emes }}$ Journées internationales d'Analyse statistique des Données Textuelles, 12-14 mars 2008, Lyon, France (http:// lexicometrica.univ-paris3.fr/jadt/jadt2008/tocJADT2008.htm)

Minvielle M., Page C., Ceron J.-P., Besson F. (2015) Extension of the SIM Reanalysis by Combination of Observations and Statistical Downscalin. Engineering Geology for Society and Territory, doi10.1007/978-3-319-09300-0_36, 2015. 1 : 189-192 